\title{
Erratum
}

\section{Precession-nutation procedures consistent with IAU 2006 resolutions}

\author{
P. T. Wallace ${ }^{1}$ and N. Capitaine ${ }^{2}$ \\ 1 Space Science and Technology Department, CCLRC/Rutherford Appleton Laboratory, UK \\ e-mail: P.T.Wallace@rl.ac.uk \\ 2 Observatoire de Paris, SYRTE/UMR 8630-CNRS, 61 avenue de l'Observatoire, 75014 Paris, France \\ e-mail: n.capitaine@obspm.fr
}

A\&A 459, 981-985 (2006), DOI: 10.1051/0004-6361:20065897

Key words. astrometry - reference systems - ephemerides - celestial mechanics - time - errata, addenda

Equations (A.1), (A.15), (A.27) and (A.36), published only in the electronic version of the article entitled "Precession-nutation procedures consistent with IAU 2006 resolutions" by P. T. Wallace and N. Capitaine, were incorrectly typeset. They should read as follows:

$\mathrm{UTC}=2006$ January $15,21^{\mathrm{h}} 24^{\mathrm{m}} 37.5$

$\mathrm{ERA}=76^{\circ} .265431053522 \equiv 5^{\mathrm{h}} 05^{\mathrm{m}} 03^{\mathrm{s}} .703452845$

$\mathrm{GST}=05^{\mathrm{h}} 05^{\mathrm{m}} 22^{\mathrm{s}} \cdot 213252581$

$\mathrm{EO}=-277^{\prime \prime} .646995746, \quad \mathrm{GST}=05^{\mathrm{h}} 05^{\mathrm{m}} 22^{\mathrm{s}} .213252562$ 